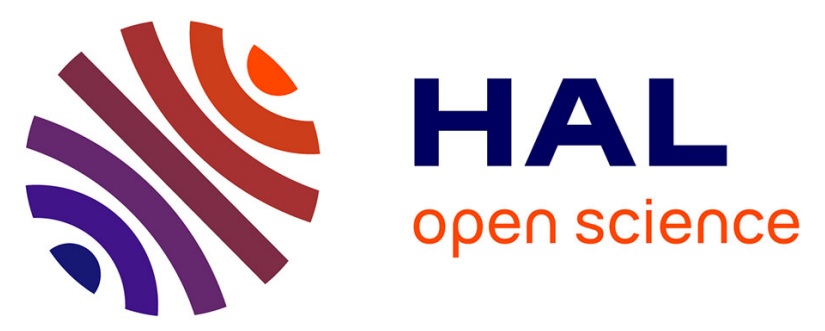

\title{
Huge Fluctuations in Weight Measurements at the Bottom of a Two-Dimensional Vertical Sheet of Grains
}

Bruno Gilles, Emmanuel Lévêque, Claude Laroche, Christophe Coste

\section{To cite this version:}

Bruno Gilles, Emmanuel Lévêque, Claude Laroche, Christophe Coste. Huge Fluctuations in Weight Measurements at the Bottom of a Two-Dimensional Vertical Sheet of Grains. Physical Review Letters, 2004, 92 (20), pp.204301. 10.1103/PhysRevLett.92.204301 . hal-01406721

\section{HAL Id: hal-01406721 \\ https://hal-univ-paris.archives-ouvertes.fr/hal-01406721}

Submitted on 1 Dec 2016

HAL is a multi-disciplinary open access archive for the deposit and dissemination of scientific research documents, whether they are published or not. The documents may come from teaching and research institutions in France or abroad, or from public or private research centers.
L'archive ouverte pluridisciplinaire HAL, est destinée au dépôt et à la diffusion de documents scientifiques de niveau recherche, publiés ou non, émanant des établissements d'enseignement et de recherche français ou étrangers, des laboratoires publics ou privés.

\section{(c)(1)}

Distributed under a Creative Commons Attribution| 4.0 International License 


\title{
Huge Fluctuations in Weight Measurements at the Bottom of a Two-Dimensional Vertical Sheet of Grains
}

\author{
Bruno Gilles \\ Unité 556, INSERM, Université Lyon I, 151 cours Albert Thomas, 69424 Lyon CEDEX 03, France \\ Emmanuel Lévêque and Claude Laroche \\ Laboratoire de Physique, CNRS, ENS Lyon, 46 Allée d'Italie 69364 Lyon CEDEX 07, France \\ Christophe Coste \\ Groupe de Physique des Solides, 2 Place Jussieu, 75251 Paris CEDEX 05, France \\ (Received 16 May 2003; revised manuscript received 19 December 2003; published 19 May 2004)
}

\begin{abstract}
Weight measurements at the bottom of a quasi-2D vertical sheet of static cohesionless grains are carried out. The grains are held between two coaxial cylinders. This peculiar setup allows us to set either periodic or fixed lateral boundary conditions. Huge relative fluctuations in weight measurements appear in case of fixed lateral walls. This may be related to some indetermination in the mobilization state of friction forces on lateral walls. This argument would hold for any piling, but would lead to huge fluctuations in 2D systems only, because of averaging effects in 3D.
\end{abstract}

DOI: 10.1103/PhysRevLett.92.204301

PACS numbers: 45.70.Cc, 05.40.-a, 46.55.+d

Granular material exhibits peculiar mechanical behavior, which underlies the complexity of the force distribution between grains $[1,2]$. In a 3D silo, the geometry of the force network is responsible for the frictional transfer of a significant amount of the vertical stress to the confining (lateral) walls. A saturation of the weight supported by the bottom plate of the silo is then observed as the height of the piling increases. This phenomenon has motivated many studies [3-6] since the seminal works of Janssen and Rayleigh [7,8], and the silo is often considered as a basic configuration that allows one to test some aspects of existing theoretical models. Recently, numerical simulations [9] have studied the transition from 2D to 3D granular packings, and have shown the limitations of quasi-2D experimental packings for the determination of purely 2D system properties.

In our study, the focus is on a 2D static sheet of grains held between two coaxial vertical cylinders. This special quasi-2D silo allows us to fix either periodic or fixed lateral boundary conditions on the same system, and then identify the specific role of lateral boundaries in the mechanical properties of the system. Particular interest is taken here in the pressure, i.e., averaged vertical stress per unit length, at the bottom of the piling. Significant differences with the 3D silo case are observed, especially concerning the apparition of huge relative fluctuations in weight measurements when grains are held between fixed lateral walls.

The experimental apparatus is described in Fig. 1. The grains are poured uniformly from the top into the gap between the cylinders. Following the reference experiment of Clément and Vanel [10], the bottom ring is then slightly lowered (over a few ten microns) in order to relax the elastic energy accumulated by the weight sensor during the loading process, and to avoid strong dependency on the filling procedure. Each pressure measurement corresponds to a new piling obtained following this protocol. We measure the apparent weight at the bottom as a function of the real mass of grains. Fixed lateral boundary conditions are considered by inserting and clamping two vertical steel sticks between the cylinders before pouring the grains (Fig. 1). These sticks will be referred to as lateral walls or boundaries. Without lateral walls, our 2D system exhibits periodic boundary conditions. In this configuration, the weight sensor at the bottom of the piling should measure the total weight of grains. In practice, some weight is lost due to friction forces between grains and the confining cylinders. However, it will be shown that this effect can be exactly quantified. Weight measurements in the periodic configuration will then provide a useful reference when considering fixed lateral walls; this is the peculiar benefit of this cylindrical geometry. Various distances between lateral walls will be examined.

At maximum filling, one can deduce from the real mass of grains, the mass per unit volume. The averaged packing fraction $C_{\infty}$ of our material can be deduced by taking into account empty space on the edge of pilings [11]. For all packings, in each configuration, we obtained an almost constant value $C_{\infty} \approx 0.90 \pm$ 0.03 . This value is greater than the random close packing fraction and is close to the largest possible value $C_{\mathrm{FCC}}(\infty)=0.907$, corresponding to a perfectly ordered close packing (triangular lattice). This indicates that our pilings are essentially regular ones, as expected in 2D lattices of identical disks. Assuming a uniform packing 


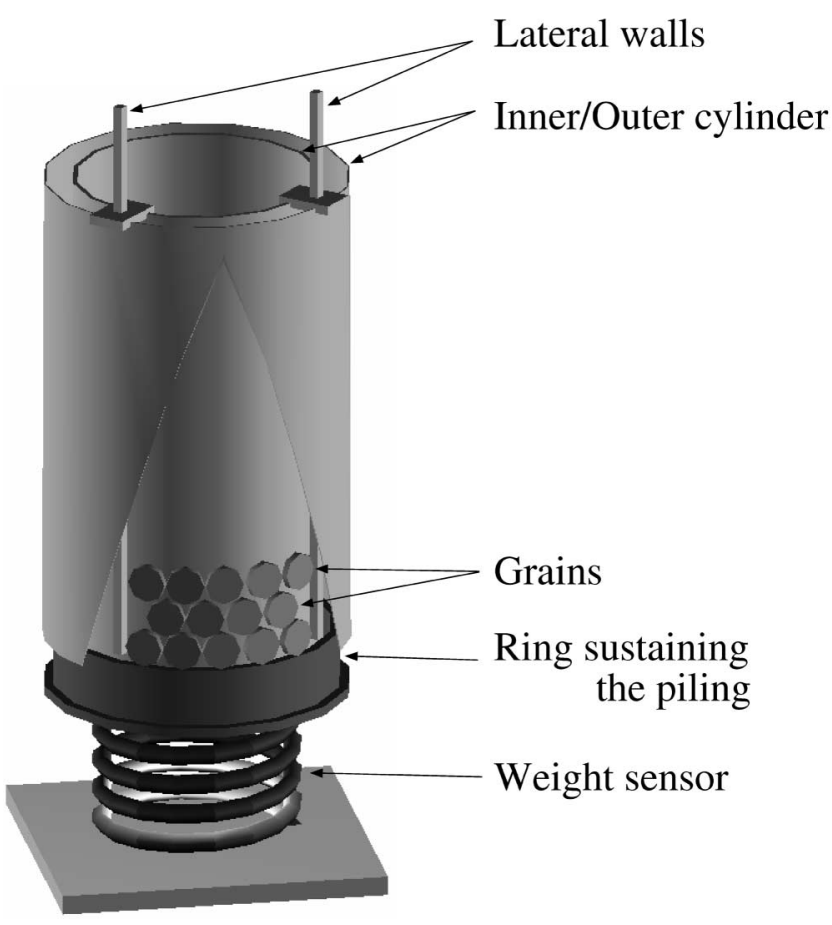

FIG. 1. Experimental setup. Grains are brass disks of radius $4 \mathrm{~mm}$ and thickness $3 \mathrm{~mm}$, confined between two coaxial, $1200 \mathrm{~mm}$ height, steel cylinders of respective radii 41.5 and $45 \mathrm{~mm}$. The circumference of the annular gap is slightly greater than 34 grain diameters. The piling lies on a ring that can move vertically, without touching the cylinders. This ring is attached to a weight sensor, measuring the apparent weight at the bottom of the piling. Two vertical steel sticks can be added, acting as lateral walls of variable positions.

fraction allows us to consider the pressure $p(z)$ at the bottom of the piling as a function of the height $z$. These variables are more adapted than the apparent and real masses when comparing systems of various widths, and are used henceforth.

Let us first consider the periodic configuration. In this case, pressure measurements are well reproducible from one piling to the other. Results are shown in Fig. 2. As $z$ increases, $p(z)$ becomes significantly lower than the whole weight (per unit length) of the piling. This indicates a significant loss of weight due to friction on confining cylinders. This effect, here reinforced by the curvature of the sheet, is actually always present in $2 \mathrm{D}$ pilings. However, in the present case, a simple geometrical model will allow us to precisely take it into account.

The case of fixed lateral walls is extremely different. We consider four different distances between lateral walls: $L$ (30 disks), $L / 2$ (15 disks), $L / 3$ (11 disks), and $L / 4$ ( 8 disks), where $L$ is the circumference (minus the width of lateral walls). Measurements for the width $L$ are presented on Fig. 2. The pressure is always lower than in the periodic configuration (for a given $z$ ), indicating an additional loss of weight due to the presence of lateral walls. The most striking point lies in the observation of huge fluctuations in the measurements when the pressure

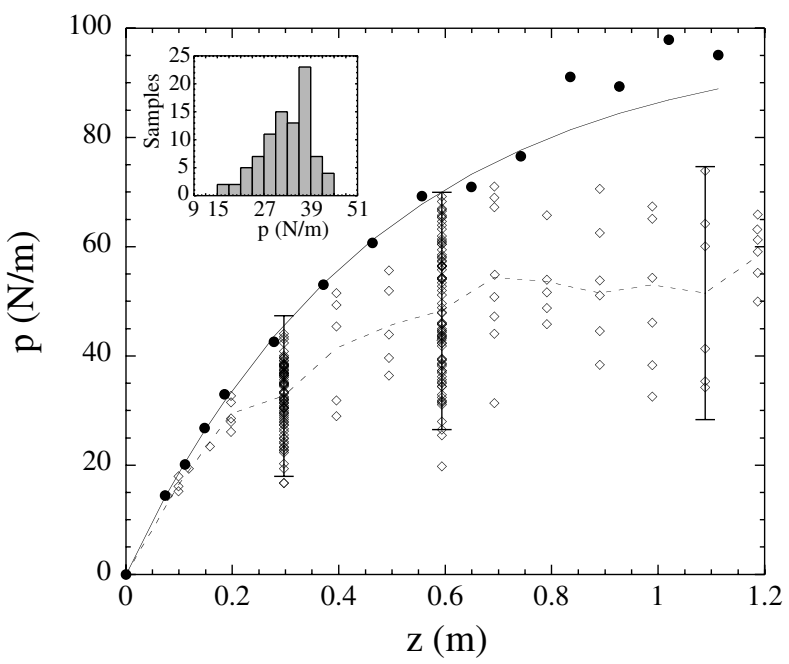

FIG. 2. Pressure $p(z)$ at the bottom of the piling for both periodic $(\bigcirc)$ and fixed $(\diamond)$ boundary conditions with distance $L$ between walls (about 30 disk diameters). The solid curve is given by Eq. (3). The mean behavior is shown by the dashed line. The inset shows the histogram of pressures obtained at $z=0.3 \mathrm{~m}$ with 100 measurements.

starts to saturate: This is the key point of our study. For $z=0.3 \mathrm{~m}$ and $z=0.6 \mathrm{~m}, 100$ independent experiments have been carried out for a system of width $L$. The histogram of pressure measurements is displayed in Fig. 2. Data are widely distributed. The values typically lie within $\pm 40 \%$ of the mean (see fluctuation bars in Fig. 2). Careful attention has been paid to the experimental conditions; these fluctuations are not related to external factors such as temperature variations or mechanical vibrations. Such a level of pressure fluctuations was not observed for 3D silos when following the same protocol [10], i.e., pouring grains from the top and lowering the piling before measurement. In this case, only few-percent relative fluctuations had been observed. A possible explanation for this difference, related to the friction mobilization state on lateral walls, will be given later.

In order to compare behaviors of the system for different widths, we plot in Fig. 3 the region containing roughly $80 \%$ of all data points around the "mean" behavior, for each width. We obtain four regions filled with different types of hatches. Regions corresponding to the widths $L$, $L / 2$, and $L / 3$ mainly overlap, while the region for $L / 4$ is significantly below. When expressing the width as a number of grains, this means that no clear evolution of the pressure occurs when reducing the width from 30 to 11 grains, while a $30 \%$ drop is observed when the width changes from 11 to 8 grains. This might be connected to a characteristic length in the system, which value would be around 10 grain diameters. Interestingly, this value is close to correlation lengths in 2D or 3D static granular pilings already mentioned in the literature [12-14].

Our motivation is now to understand, at least qualitatively, the observed level of fluctuations in the presence of 


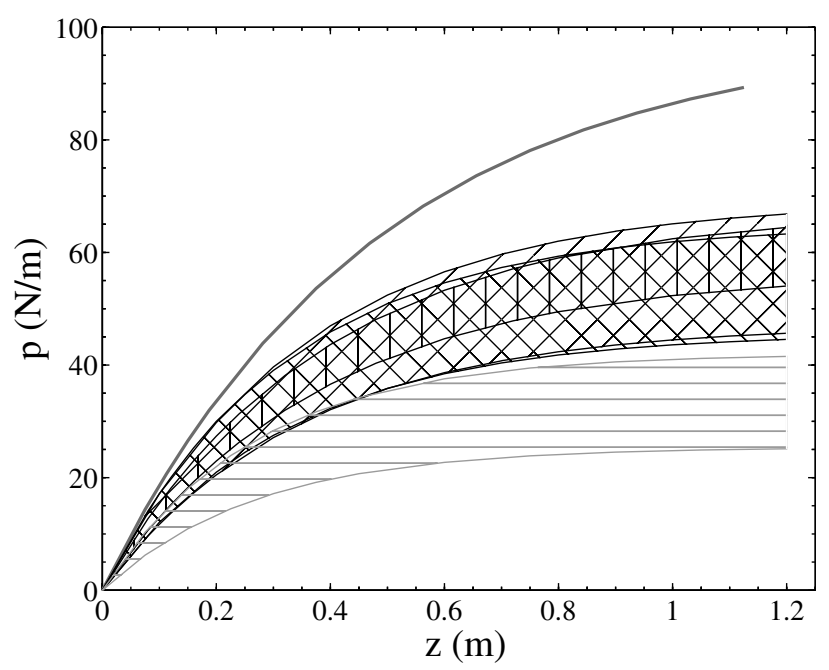

FIG. 3. Plot of regions containing roughly $80 \%$ of the data around the mean, for each distance between lateral walls: $L$ (right-slanting lines), $L / 2$ (left-slanting lines) $L / 3$ (vertical lines) and $L / 4$ (horizontal lines). The model for the periodic case is reported for reference (upper bold line).

fixed lateral boundary conditions. A first step is to describe the simpler case of periodic boundary conditions.

As previously mentioned, our pilings are essentially regular and may then be modeled as perfect triangular lattices of disks lying on the inside of a tube. The effect of the inner cylinder is neglected since horizontal forces are mainly centrifugal. The curvature of the sheet produces a nonzero normal component for the contact force between the outer cylinder and one grain. This induces a friction force $F_{\mu}$, which is assumed to be at the limit of sliding friction and oriented in the upward direction (due to the lowering process [10]). Let us denote $F$ the resulting force applied by a disk on both its underneath neighbors (see Fig. 4). Conditions of equilibrium for a disk, combined with the hypothesis of the limit of sliding friction leads to the following expression for the friction force $F_{\mu}$ (in the limit of small curvature):

$$
F_{\mu}=(\alpha / \sqrt{3}) \mu_{\mathrm{c}} F,
$$

where $\alpha$ is the angle between the plane containing the
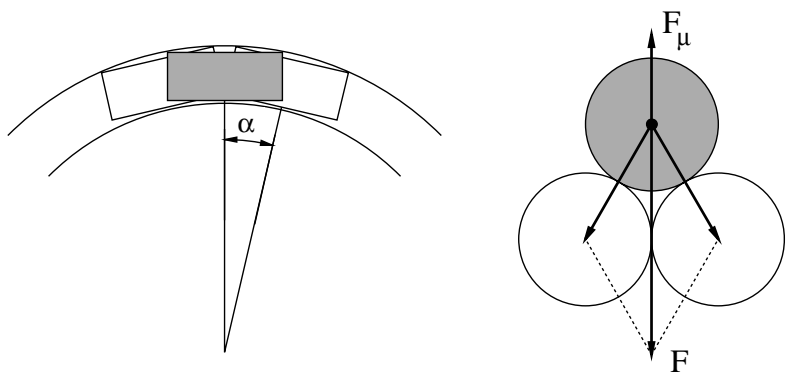

FIG. 4. Configuration of grains considered in the modeling of friction against cylinders. For convenience, grains are presented in the same plane on the right scheme. disk and the plane containing one of its underneath neighbors. $\mu_{\mathrm{c}}$ stands for the friction coefficient between the disks and the inner wall of the outer cylinder (Fig. 4). In our configuration, $\alpha=95 \times 10^{-3}$ rad. A direct measurement of $\mu_{\mathrm{c}}$ gives $\mu_{\mathrm{c}} \simeq 0.31$. Note that friction between grains has been ignored in this calculation.

Using Eq. (1), a Janssen-like approach [7,15] leads to

$$
d p / d z=\rho g-p / z^{*} \quad \text { where } z^{*} \equiv 2 \sqrt{3} R /\left(\alpha \mu_{\mathrm{c}}\right) .
$$

Here, $p(z) \approx F /(2 R)(\alpha \ll 1)$ is the pressure acting on the layer of grains at height $z, R$ is the radius of the disks, $\rho$ is the mass per unit volume of grains, and $g$ is the acceleration of gravity. The coordinate $z$ is taken along a downward vertical axis, with the origin at the top of the piling. At $z=0$ the pressure is null. The integration of Eq. (2) gives the pressure profile

$$
p(z)=\rho g z^{*}\left(1-e^{-z / z^{*}}\right) .
$$

Figure 2 shows that Eq. (3) describes very well, without any adjustable parameter, the experimental data $(0)$ in the periodic boundary configuration.

Let us now consider the fixed boundary case. Following Janssen's approach, Pitman [5] has suggested to relate pressure fluctuations at the bottom of a 3D silo, to randomness of friction coefficients. However, few-percent fluctuations of grain-grain or grain-wall friction give only few-percent fluctuations of the pressure. Here, we introduce randomness in the mobilization state of friction forces between grains and lateral walls: We assume that friction forces are fully mobilized (at the limit of sliding) but can be directed either upward or downward. In Ref. [10], it is argued that lowering the piling ensures upward fully mobilized friction all over the boundaries of the system. This argument relies on the picture of a solid downward displacement of the piling. However, the lowering process is also accompanied by rotation of individual grains. These rotational degrees of freedom are necessarily frustrated in our triangular lattice [16], which results in some indetermination in the friction direction at lateral boundaries. We thus propose that the high dispersion of our measurements may be related to randomness in the direction of friction at lateral boundaries.

The situation concerning the direction of friction forces against the confining cylinders is different. Indeed, the resulting contact force between a disk and the outer cylinder is independent of the rotation direction of the disk, and is oriented upward provided there is sliding downward during the lowering process. Thus, random rotation directions do not yield indetermination in friction forces between disks and the outer cylinder. This point justifies the form of Eq. (2), where we assumed a fully mobilized friction force oriented upward.

We now propose to extend the previous Janssen-like model Eq. (2) by adding a contribution due to friction against lateral walls. The direction of these friction forces is considered as random. The equation for the pressure 


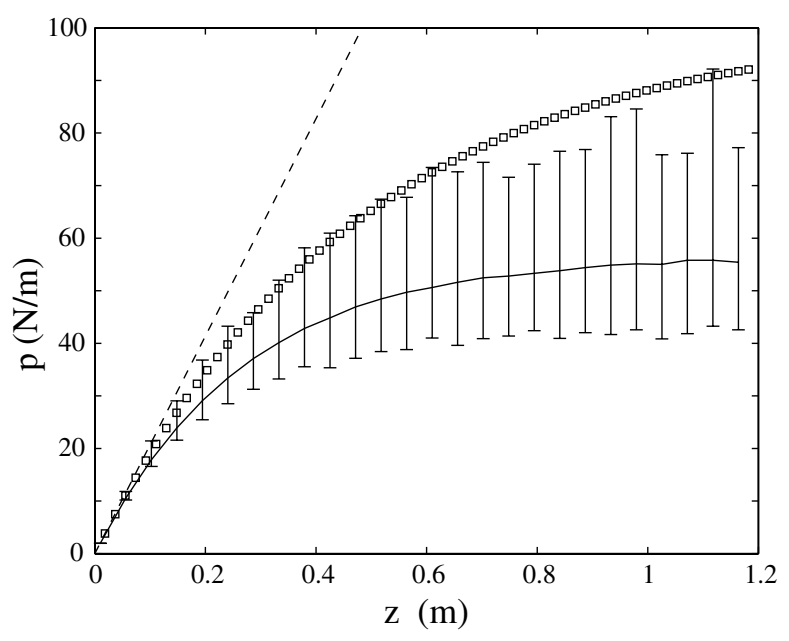

FIG. 5. Modified Janssen model defined by Eq. (4) with $\kappa=$ 0.725 and $\mu_{\text {wall }}=0.6$ for a system of width $L$ (30 disks). Results for the periodic case are given by $(\square)$. The dashed line represents the hydrodynamical law $p(z)=\rho g z$. The error bars indicate the range of fluctuations around the mean value (solid line) for 1000 simulations at a given height.

$p(z)$ at height $z$ of a $N$-disk-wide piling reads

$$
d p / d z=\rho g-\left[1 / z^{*}+\kappa \mu_{\mathrm{wall}} \epsilon(z) /(2 N R)\right] p,
$$

where $\kappa$ is the Janssen's redirection coefficient, $\mu_{\text {wall }}$ is the friction coefficient between disks and lateral walls, and $\epsilon(z)$ is a random variable taking values \pm 1 , indicating the direction of friction for the slice $z$. This equation can be integrated numerically to get the bottom pressure, provided that statistics of the stochastic process $\epsilon(z)$ are given. For each $z$, a thousand numerical experiments have been performed. Results are displayed in Fig. 5 for an uncorrelated process in $z$ with probability $P(\epsilon=1)=$ 0.25 (downward) and $P(\epsilon=-1)=0.75$ (upward). This simple probability distribution takes into account indetermination in the direction of friction forces, and is consistent with the mean friction being oriented upward. These results should be compared to Fig. 2. We observe that randomness in the direction of friction at lateral walls conducts to a fluctuation level comparable to that of the experimental data. This basic ingredient is thus sufficient to explain the dispersion of our measurements.

Our argument could be invoked for any confined granular piling, since direction of friction forces at individual grain-boundary contacts is a priori unpredictable. However, in 3D silos it would lead to a lower fluctuation level. Indeed, the effective direction of friction for a layer would now reflect the mean direction over all grains at the circumference of the layer. In practice, this mean direction does not fluctuate much because averaging is made over a large number of grains. Consequently, the pressure at the bottom plate does not fluctuate much as well. This argument is consistent with some recent mea- surements indicating that the variance of pressure fluctuations at the bottom of a 3D silo is inversely proportional to the number of grains at the circumference of a layer [17]. In 2D, the averaging process is made over only two grains. It thus induces much larger pressure fluctuations.

In conclusion, this experiment points out the major role played by friction forces against walls, in the mechanical properties of a confined 2D granular medium. More precisely, indetermination in the direction of the friction force at individual grain-wall contact is responsible for huge fluctuations in the apparent weight at the bottom of a 2D silo. We give a basic model which reproduces this level of fluctuations, just considering randomly distributed direction of friction forces against (lateral) walls. This indetermination is emphasized here because of the quasiregular structure of our pilings, which strengthens frustration of rotational degrees of freedom of grains. Nevertheless, indetermination of the mobilization state of friction forces at the walls a priori holds for any piling, regardless of its microscopic structure. It is all the more important, since forces at the walls are very close to incipient failure [9]. In 3D, the effect of this indetermination is weakened since friction force acting on a layer of the piling is averaged over a large number of grains.

We thank L. Vanel and E. Falcon for useful discussions.

[1] H. M. Jaeger, S. R. Nagel, and R. P. Behringer, Rev. Mod. Phys. 68, 1259 (1996).

[2] T. Travers et al., Europhys. Lett. 4, 329 (1987).

[3] S. N. Coopersmith et al., Phys. Rev. E 53, 4673 (1996).

[4] R. Peralta-Fabi, C. Málaga, and R. Rechtman, Europhys. Lett. 45, 76 (1999).

[5] E. B. Pitman, Phys. Rev. E 57, 3170 (1998).

[6] L. Vanel et al., Phys. Rev. Lett. 84, 1439 (2000).

[7] H. A. Janssen, Z. Ver. Deutscher Ing. 39, 1045 (1895).

[8] Lord Rayleigh, Philos. Mag. 36, 11 (1906); 36, 61 (1906); 36, 129 (1906); 36, 206 (1906).

[9] J.-W. Landry, G.-S. Grest, and S.-J. Plimpton, cond-mat/ 0302115.

[10] L. Vanel and E. Clément, Eur. Phys. J. B 11, 525 (1999).

[11] It can be done by writing $C_{\infty} \equiv C_{\text {measured }}(L) \times$ $\left[C_{\mathrm{FCC}}(\infty)\right] /\left[C_{\mathrm{FCC}}(L)\right]$, where $C_{\mathrm{FCC}}(L)$ is the packing fraction of a regular triangular lattice of width $L$, and can be easily computed.

[12] P. G. de Gennes, Rev. Mod. Phys. 71, 374 (1999).

[13] S. Ouaguenouni and J.-N. Roux, Europhys. Lett. 39, 117 (1997).

[14] F. Radjai, M. Jean, J.-J. Moreau, and S. Roux, Phys. Rev. Lett. 77, 274 (1996).

[15] R. M. Nedermann, Statics and Kinematics of Granular Materials (Cambridge University Press, Cambridge, England, 1992).

[16] L. M. Schwartz, D. L. Johnson, and S. Feng, Phys. Rev. Lett. 52, 831 (1984).

[17] L. Vanel and E. Clément (private communication). 